\title{
FLORÍSTICA E ESTRUTURA DA COMUNIDADE HERBÁCEA E ARBUSTIVA DA PRAIA DO FERRUGEM, GAROPABA-SC. ${ }^{1}$
}

\author{
Elisabeth Danilevicz ${ }^{2}$ \\ Heidi Janke ${ }^{2}$ \\ Lúcia Helena S. Pankowski ${ }^{2}$
}

RESUMO - A Praia do Ferrugem, constituída por sedimentos do Quartenário e afloramentos do Complexo Cristalino, localiza-se no litoral sul de Santa Catarina, Município de Garopaba. Nesta praia, foi realizada uma análise da vegetação de dunas em outubro de 1989. Um total de 50 unidades amostrais, na forma de quadrados com área de $1 \mathrm{~m}^{2}$ cada, foram distribuídas sistemático-aleatoriamente na vegetação herbácea. Foi registrada a cobertura das espécies (escala de Daubenmire por Bailey \& Poulton). Devido a ocorrência de formaçōes arbustivas, foram feitos registros de presença de espécies e estrutura desta comunidade distinta. São apresentadas descriçōes dos diversos habitats e suas espécies dominantes com base nos parâmetros fitossociológicos, listagem das espécies e respectivas formações vegetais, bem como diagrama de perfil de uma formação arbustiva típica.

Palavras-chave: restinga, dunas costeiras, vegetação, flora, Santa Catarina.

ABSTRACT - Ferrugem Beach is made up of Quaternary sediments and Crystaline Complex outcrops which are present on the southern coast of Santa Catarina State, in the town of Garopaba. A survey of the sand dune vegetation was carried out in Oftober 1989. Fifty quadrats, $1 \mathrm{~m}^{2}$ in area, were sampled in a random-systematic arrangement in the herbaceous vegetation. The species cover was recorded according to Daubenmire's cover scale. Species presence was recorted in the shrubby vegetation. The various habitats are described, together with dominant species based on phytosociological data. $A$ check-list of the species is given as well as a diagrammatic profile of a typical shrub thicket.

Key-words: "restinga", coastal dune, vegetation, flora, Santa Catarina.

\section{Introdução}

O litoral sudeste brasileiro, ou das escarpas cristalinas, forma uma ampla

1 - Trabalho realizado com o apoio da Associação dos Amigos da Praia da Barra, Garopaba-SC.

2 - Projeto Praia do Ferrugem - Rua: Lima e Silva 861/705 - CEP 90050 - Porto Alegre-RS. 
concavidade entre o sul do Espírito Santo e o Cabo de Santa Marta, Santa Catarina; caracteriza-se pelas frentes das escarpas de rochas do Complexo cristalino Pré-Cambriano entremeadas por deposições marinhas do Quartenário (Suguio \& Tessler, 1984).

Á vegetação do litoral catarinense possui várias formações, todas sob efeito patente dos agentes geológicos (Reitz, 1961). A formação vegetacional de restinga encontra-se sobre depósitos marinhos apresentados sob forma de línguas de areia, fechando enseadas ou separando uma laguna do oceano (Lacerda et al., 1982). A diversidade topográfica e ambiental da restinga proporciona a formação de muitos habitats com grande variedade de comunidades vegetais e "de uma flora rica e variada" (Araújo, 1984).

Devido ao grande interesse turístico e de lazer, a orla marítima vem sendo constantemente degradada (Soares, 1984), ocasionando alterações à diversidade específica e à qualidade ambiental. $\mathrm{O}$ interesse na proteção da qualidade de vida e ambiental na Praia do Ferrugem motivou a Associação dos Amigos da Praia da Barra, entidade sem fins lucrativos que congrega veranistas e moradores da região em apreço, a solicitar um estudo sobre a preservação da área, incluso este. O fundamento deste trabalho está na intenção de descrever a vegetação da praia ameaçada pela especulação imobiliária, fornecendo subsídios para uma recomposição da paisagem no caso de destruição do complexo de dunas.

\section{Material e Métodos}

A Praia do Ferrugem (Figura 1) constitui uma baía preenchida por sedimentos oriundos principalmente da plataforma continental granítico-gnáissica submergida (Justus et al.. 1986) 


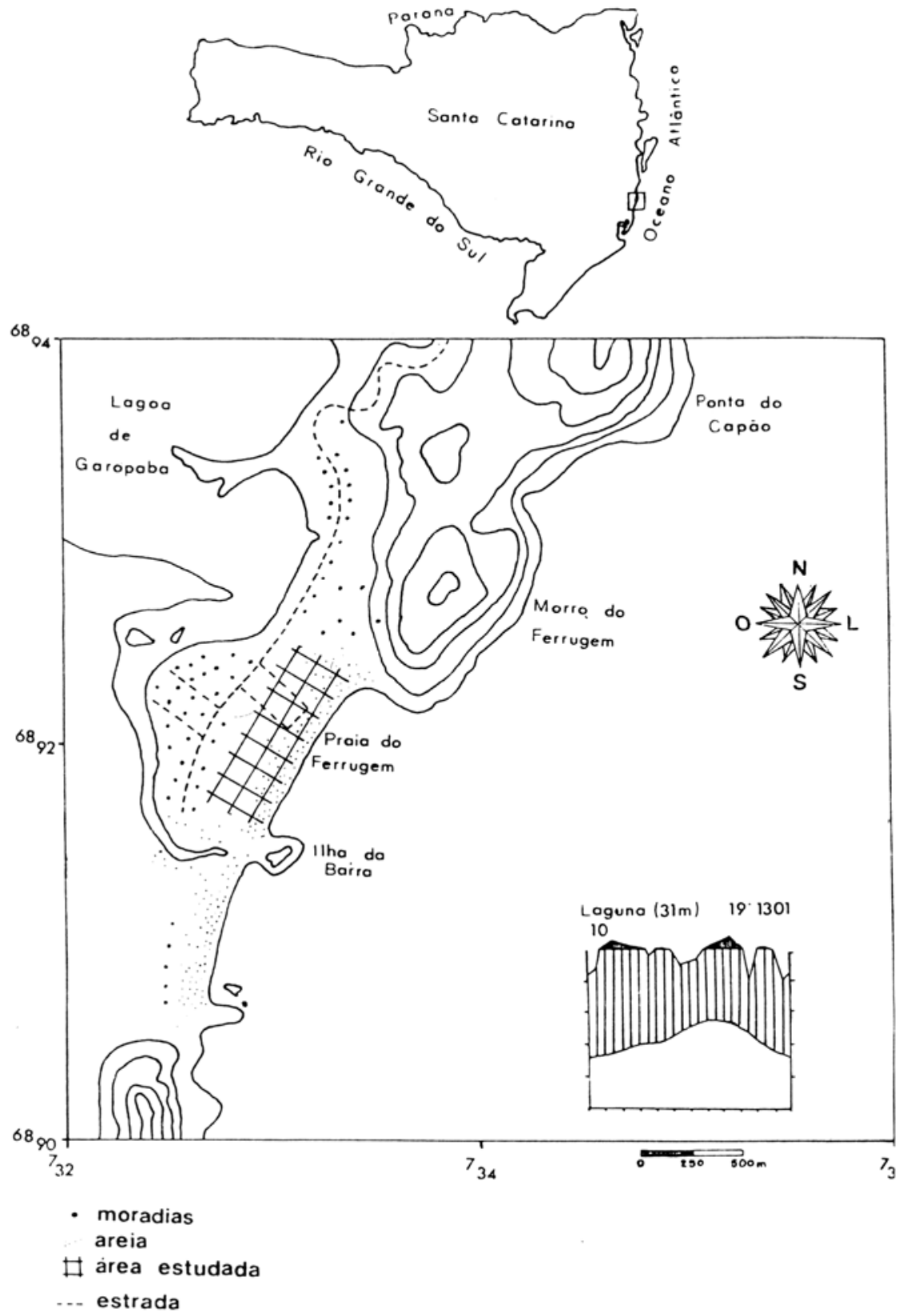

Figura 1 - Localização da Praia do Ferrugem, Garopaba, SC. 
Classificado como subtropical úmido, o clima da região litorânea catarinense apresenta suave aumento da tropicalidade de sul para norte (Pfadenhauer \& Butzke, 1978).

Para fins de reconhecimento florístico e estrutural da comunidade, foram definidas duas subunidades básicas de vegetação litorânea: a vegetação herbáœa e formações de vegetação arbustiva.

A vegetação herbácea é dominante na área e ocorre a partir da zona de maré (região mediana entre as horas de máxima amplitude, preamar, e mínima amplitude, baixa mar) até o ponto onde as dunas, ou cômoros, desaparecem. Nesta extensão, aproximadamente $110.000 \mathrm{~m}^{2}$ (Figura 1), foram estabelecidas, a cada 100 metros, transecções perpendiculares ao mar. Devido a terraplenagem e aterro que descaracterizaram uma porção da área, foi necessário um intervalo de, aproximadamente 150 metros, entre as transecções limítrofes a este local. As unidades amostrais foram distribuídas nas transecções de forma sistemático-aleatória, com intervalos de 20 metros. O amostrador adotado possuía área de $1 \mathrm{~m}^{2}$ e forma quadrangular. A amostragem totalizou 50 unidades amostrais, em cada qual foi registrada a cobertura das espécies ocorrentes, segundo a escala de Daubenmire modificada por Bailey \& Poulton (apud Müller-Dombois \& Ellenberg, 1974). Nestas foram também observados alguns fatores abióticos.

A partir de dados de campo foram calculados os seguintes parâmetros fitossociológicos: freqüência absoluta e relativa, cobertura absoluta e relativa, densidade absoluta e relativa e o índice de valor de importância (IVI). A ocorrência das 10 espécies de maior IVI foi plotada no mapa da área para análise da distribuição das espécies ao longo do local estudado. A partir desta distribuição e observação das espécies acompanhantes foi elaborado o mapa de distribuição dos tipos de vegetação, segundo Waechter (1985). A área alterada também foi mapeada.

A denominação "formações arbustivas" foi atribuída aos agrupamentos de vegetação onde os arbustos são predominantes. Foram levantadas as espécies ocorrentes e, para a construção do diagrama de perfil foram observadas a estratificação e dimensões das espécies em um transecto de $1 \mathrm{~m}$ de largura na borba de um agrupamento típico.

Para as espécies não identificadas a campo, foram coletadas amostras objetivando posterior identificação.

As exsicatas estão depositadas no herbário Prof. Dr. Alarich Schultz (HAS) da Fundação Zoobotânica do Rio Grande do Sul sob os números 28.197 à 28.288.

\section{Resultados e discussão}

Foram identificadas 135 espécies, distribuídas em 51 famílias botânicas 
(Tabela 1). Um total de $51,85 \%$ destas espécies pertencem exclusivamente a vegetação herbácea de restinga, outros $40,00 \%$ exclusivamente às formações arbustivas e apenas $8,15 \%$ ocorrem simultaneamente em ambas formaçōes. Das 81 espécies que ocorrem na comunidade herbácea $(\mathrm{CH}), 66$ foram amostradas (Tabela 1).

Em sua maioria, as espécies observadas correspondem às formações típicas de dunas do Brasil meridional (Reitz, 1961; Rambo, 1954; Pfadenhauer \& Ramos, 1979; Soares, 1984; Waechter, 1985 e Cordazzo \& Seeliger, 1988). Ainda foi constatada a ocorrência de espécies tropicais como Ipomoea pes-caprae, Canavalia rosea, Mariscus pedunculatus e Scaevola plumieri que encontram seu limite austral no litoral catarinense (Reitz, 1961; Cordazzo \& Seeliger, 1988). Este limite demonstra a transição biogeográfica entre as regiões setentrional tropical e meridional temperada fria, que tem lugar no extremo sul do Brasil e Uruguai (Cordazzo \& Seeliger, 1988).

A vegetação herbácea alcança cobertura média de $30,84 \%$ da superfície do solo amostrado, tendo altura média estimada em 15 centímetros.

A Tabela 2 apresenta parâmetros fitossociológicos das espécies com índice de valor de importância superior a cinco, agrupando as demais espécies e seus valores. A maioria das espécies possui baixa densidade e baixo grau de cobertura, evidenciando uma descontínua distribuição. Entretanto, Hydroco'tyle bonariensis, Mariscus pedunculatus e Polygala cyparissias estão regularmente distribuídas ao longo da área amostrada, demonstrando alta freqüência na fisionomia da vegetação.

A vegetação halófila das dunas frontais (Figura 2) é composta principalmente por Spartina ciliata, Senecio crassiflorus, Hydrocotyle bonariensis e Mariscus pedunculatus. Foram também registrados Blutaparon portulacoides e Alternanthera maritima, porém fora das unidades amostrais. Enquadram-se no tipo de vegetação pioneira halófila (Waechter, 1985), parecendo serem espécies características deste ambiente (Cordazzo \& Costa, 1989).

$\mathrm{Na}$ porção sudoeste da área (próximo ao canal da Lagoa de Garopaba) ocorrem as dunas mais elevadas, com aproximadamente 7 metros de altura. Neste local a vegetação psamófila das dunas móveis (Figura 2) é representada principalmente por Smilax campestris, Mariscus pedunculatus e Polygala cyparissias, com uma ocorrência alternada de Spartina ciliata em áreas mais próximas ao mar e Androtrichum trigynum nas dunas mais internas. Esta última espécie possui ainda ampla distribuição nas dunas estabilizadas e baixadas úmidas, apresentando redução da população nas dunas frontais (Soares, 1984).

Logo após as dunas frontais, onde o desnível do terreno é menos acentuado, ocorrem pequenas dunas, quase planas, e baixadas úmidas. Nas porções mais elevadas, a vegetação psamófila das dunas estáveis (Figura 2) ocorre de forma bastante uniforme em altura e distribuição espacial das espécies. São características destes locais: Porophyllum ruderale, Hydrocotyle bonariensis, 
Ipomoea pes-caprae, Polygala cyparissias, Acycarpha spatulata, Mariscus pedunculatus e Oxypetalum tomentosum, associadas à Dodonaea viscosa, que possui dimensões muito reduzidas e está bem integrada à comunidade herbácea das dunas estáveis. Nas depressões úmidas entre cordões litorâneos, também denominadas "brejos herbáceos" (Araújo \& Henriques, 1984), a vegetação psamófila das baixadas úmidas (Figura 2) é representada principalmente por Hydrocotyle bonariensis, Androtrychum trigynum, Centella asiatica e Imperata brasiliensis. Androtrychum trigynum tem sua distribuição relacionada ao grau de umidade no solo (Pfadenhauer \& Ramos, 1979), sendo sua maior freqüência nas baixadas úmidas.

Algumas destas baixadas úmidas apresentam locais com incidência de Drosera brevifolia, Cyperus obtusatus e Cyperus consanguineos, também espécies hidrófilas.

As formações arbustivas (Figura 3) possuem uma composição semelhante às matas de restinga, entretanto com porte bem reduzido. Os arbustos formam a estrutura espacial do conjunto, propiciando um ambiente favorável ao desenvolvimento de ervas terrestres e epifíticas. Alguns destes arbustos são espécies que, em outras condições ambientais assumem porte arbóreo, como por exemplo, Guapira opposita, Rapanea parvifolia, Gomidesia palustris e Tabebuia pulcherrima.

As dimensōes destas formações são variáveis. As menores assumem formas circulares e ovaladas e as de maior dimensão são mais irregulares. A altura média destas formações é de 2,5 metros e seu diâmetro máximo é variável. Sua ocorrência se dá nos cordões litorâneos arenosos ou em suas depressões. $\mathrm{Na}$ porção nordeste da praia ocorre um predomínio maior das formações arbustivas em relação à vegetação herbácea (Figura 2 ).

Os arbustos Lithraea brasiliensis, Opuntia arechevaletae, Psidium cattleyanum, Guapira opposita, Dodonaea viscosa e Tabebuia pulcherrima apresentam grande freqüência nos grupos. Já no extrato herbáceo destas formaçōes, existe um predomínio de Peperomia corcovadensis, Vriesea friburgensis e Smilax campestris. As ervas epifíticas estão representadas, principalmente, por Microgramma vaccinifolia e as trepadeiras por Smilax elastica.

\section{Agradecimentos}

À Hilda Longhi-Wagner, à Estela Chini Magri, ao Nelson Ivo Matzenbacher, ao Marcos Sobral e ao Rogério Machado Bueno pelo auxílio na determinação de algumas espécies.

Ao Jorge Luiz Waechter e a Márcia T.M.B. das Neves pela leitura do manuscrito. 


\section{Referências bibliográficas}

ARAÚJO, D.S.D. de 1984. Comunidades vegetais. In: L.D. LACERDA, et al. (Orgs.) Restingas: origem, estrutura, processos. CEUFF, Niterói. p. 157.

ARAÚJO, D.S.D. de \& R.P.B. HENRIQUES, 1984. Análise florística das restingas do Estado do Rio de Janeiro. In: L.D. LACERDA, et al.(Orgs.) Restingas: origem, estrutura, processos. CEUFF, Niterói. p. 159-193.

CORDAZZO, C.V. \& V. SEELIGER. 1988. Phenological and biogeographical aspects of coastal dune plant communities in southern Brazil. Vegetatio, 75:169-173.

CORDAZZO, C.V. \& C.S.B. COSTA. 1989. Associaçōes vegetais das dunas frontais de Garopaba (SC). Ciência e Cultura, São Paulo, 41(9):906-910.

JUSTUS, J.D.; M.L.A. MACHADO \& M.S.M. FRANCO. 1986. Geomorfologia. In: Levantamentos de recursos naturais. Rio de Janeiro, IBGE. V. 33. p. 313-404.

LACERDA, L.D.; D.S.D. ARAÚJO \& N.C. MACIEL. 1982. Restingas b rasileiras: uma bibliografia. Publ. Inst. Biol., UFRJ, Rio de Janeiro, 55p.

MUELLER-DOMBOIS, D. \& H. ELLENBERG. 1974. Aims and methods of vegetation ecology. New York, John Wiley \& Sons. 547p.

PFADENHAUER, J.S. \& A. BUTZKE. 1978. O clima da região carbonífera do Estado de Santa Catarina. In: Relatório final do convênio FATMAUFRGS. Porto Alegre, NIDECO. p. 41-54.

PFADENHAUER, J.S. \& R.F. RAMOS. 1979. Um complexo de vegetação entre dunas e pântanos próximo a Tramandaí-RS, Brasil. Iheringia, série botânica, Porto Alegre, (25):17-26.

RAMBO, B. 1954. História da flora do litoral riograndense. Sellowia. Itajaí, 6(6):113-169.

REITZ, P.R. 1961. Vegetação da zona marítima de Santa Catarina. Sellowia. Itajaí, 13(13):17-117.

SOARES, J.J. 1984. Levantamento fitossociológico de uma faixa litorânea do Rio Grande do Sul entre Tramandaí e Praia do Barco. In: L.D. LACERDA. et al. (Orgs.) Restingas: origem, estrutura, processos. CEUFF, Niterói. p. 381-394.

SUGUIO, K. \& M.G. TESSLER. 1984. Planícies de cordões litorâneos quaternários do Brasil: origem e nomenclatura. In: L.D. LACERDA, et al. (Orgs.) Restingas: origem, estrutura, processos. CEUFF, Niterói. p. 15-25.

WAECHTER, J.L. 1985. Aspectos ecológicos da vegetação de restinga no Rio Grande do Sul, Brasil. Com. Mus. Ciên. PUCRS, série Bot., Porto Alegre, (33):49-68. 
Tabela 1 - Relação das Famílias e Espécies da Restinga da Praia do Ferrugem-SC, com Hábito e Comunidade Vegetal a que pertencem.

FAMÍLIA/ESPÉCIE

HÁBITO

COMUNIDADE

AMARANTHACEAE

Alternanthera maritima (Mart.) St.-Hil.

Alternanthera monquinii (Webb. ex Moq.) Dusen

Blutaparon portulacoides (St.-Hil.) Mears

Gomphrena perennis L.

ANARCADIACEAE

Lithraea brasiliensis L. March.

ANNONACEAE

Rollinia emarginata Schlecht. AQUIFOLIACEAE

Ilex theezans Mart. ARACEAE

Anthurium gaudichaudianum Kunth ASCLEPIADACEAE

Jobinia conivens (Hook. et Arn.) Malme

* Oxypetalum tomentosum Hook. et Arn. BIGNONIACEAE

Tabebuia pulcherrima Sandw. BORAGINACEAE

Cordia verbenaceae DC. BROMELIACEAE

Aechmea gamosepala Wittmack

Bromelia antiacantha Bertol.

Dyckia maritima Backer

Tillandsia gardneri Lindl.

Tillandsia stricta Soland.

Tillandsia tenuifolia $\mathrm{L}$.

Vriesea friburgensis Mez

Vriesea platzmannii E. Morr.

CACTACEAE

Lepismium cruciforme (Vell.)Miq.

Opuntia arechevaletae Speg. ex Arech.

CALYCERACEAE

* Acicarpha spathulata R. Br.

CHENOPODIACEAE

Chenopodium retusum Juss.

COMMELINACEAE

*Floscopa sp.

$\mathrm{Et} / \mathrm{Er}$

$\mathrm{Er} / \mathrm{Ep}$

$\mathrm{Er} / \mathrm{Ep}$

* Chaptalia integerrima (Vell.) Bukart

*Conyza sp.

${ }^{*}$ Eclipta bellidioides (Spreng.) Sch.-Bip.

*Facelis retusa (Lam.) Sch.-Bip

*Gamochaeta americana (Mill.) Weddell
Er

Er

Er

Er

Ar

Ar

Ar

Er

Et

Ar

Ar

Er

Er

Er

Ep

Ep

Ep

Ep

Ar

Er

Er

Er

Er

$\mathrm{CH}$

$\mathrm{CH}$

CA

$\mathrm{CH}$

CA

CA

CA

CA

CA

CA

$\mathrm{CH} / \mathrm{CA}$

$\mathrm{CA} / \mathrm{CH}$

CA

CA

CA

CA

CA

CA

CA

CA

CA

CA

CA

$\mathrm{CH}$

$\mathrm{CH}$

$\mathrm{CH} / \mathrm{CA}$

Er

$\mathrm{CH}$

$\mathrm{CH}$

$\mathrm{CH}$

Er

$\mathrm{CH}$ 
Tabela 1: Continuação

* Gamochaeta falcata (Lam.) Cabr.

*Gamochaeta filaginea (DC.) Cabr. COMPOSITAE

*Hypochoeris variegata (Lam.) Baker

Mikania involucrata Hook. et Arn.

*Noticastrum acuminatum (DC.) Cuatrecasas

*Parthenium sp.

*Porophyllum ruderale (Jacq.) Cass.

*Senecio crassiflorus (Lam.) DC.

Senecio montevidensis (Spr.) Baker

*Sommerfeltia spinulosa (Spreng.) Less

*Symphyopappus casarettoi Robs. CONVOLVULACEAE

*Dichondra sericea Swartz

*Evolvulus glomeratus Ness et Martius

*Evolvulus pussillus Choisy

*Ipomoea pes-caprae (L.) Sweet.

*Ipomoea littoralis Boiss.

CYPERACEAE

*Androtrichum trigynum (Spreng.) Pfeiff.

*Cyperus reflexus $\mathrm{Vahl}$

Er

$\mathrm{CH}$

Er

$\mathrm{CH}$

Er

CA

$\mathrm{Er}$

$\mathrm{CH}$

Er

$\mathrm{CH}$

Er

$\mathrm{CH}$

Er

$\mathrm{CH}$

Er

$\mathrm{CH}$

Er

$\mathrm{CH}$

Er

$\mathrm{CA} / \mathrm{CH}$

*Cyperus obtusatus (Presl.) Mattf. et Kükenth.

Er

$\mathrm{CH}$

Er

$\mathrm{CH}$

Er

$\mathrm{CH}$

$\mathrm{Er}$

$\mathrm{CH}$

Er

$\mathrm{CH}$

*Cyperus consanguineos Kunth.

*Mariscus pedunculatus (R.Br.) T.Koyama

*Scirpus cernuus Vahl

DROSERACEAE

*Drosera brevifolia Pursh.

DRYOPTERIDACEAE

*Rumohra adiantiformis (Forst.) Ching EUPHORBIACEAE

*Euphorbia serpens H.B.K.

Sebastiania corniculata (Vahl) Muell. Arg. GESNERIACEAE

Codonanthe gracilis (Mart.) Hanstein

Er

$\mathrm{CA} / \mathrm{CH}$

Er

$\mathrm{CH}$

$\mathrm{Er} \quad \mathrm{CH}$

$\mathrm{Er} \quad \mathrm{CH}$

$\mathrm{Er} \quad \mathrm{CH}$

$\mathrm{Er} \quad \mathrm{CH}$

Er

$\mathrm{CH}$

Er

CA

Er

$\mathrm{CH}$

Ar

CA

Sinningia canescens (Mart.) Wiehler

Ep

CA GOODENIACEAE

Scaevola plumieri (L.) Vahl

Er

CA

GRAMINEAE

*Andropogon selloanus (Hack.) Hack.

Ar

$\mathrm{CH}$

*Axonopus sp.

Er

$\mathrm{CH}$

*Briza erecta Lam.

Er

$\mathrm{CH}$

$B$. uniolae (Nees) Nees

Er

$\mathrm{CH}$

${ }^{*}$ Cenchrus pauciflorus Bentham

Er

$\mathrm{CH}$

*Eragrostis cataclasta Nicora

Er

$\mathrm{CH}$

${ }^{*}$ E. ciliaris $(\mathrm{L}$.) R. B rown

Er

$\mathrm{CH}$

* Imperata brasiliensis Trin.

* Ischaemum minus Presl

Er

$\mathrm{CH}$

* Panicum milioides Nees ex Trin.

Er

$\mathrm{CH}$

*P. racemosum (Beauv.) Spreng.

Er

$\mathrm{CH}$

*P. sabulorum Lam.

Er

$\mathrm{CH}$

Er

$\mathrm{CH}$ 
Tabela 1: Continuação

*Panicum sp.

*Paspalum vaginatum $\mathrm{Sw}$.

*Spartina ciliata Brongn.

HYPOXIDACEAE

${ }^{*}$ Hypoxis decumbens $\mathrm{L}$.

IRIDACEAE

*Sisyrhinchium sp.

JUNCAGEAE

*Juncus capillaceus Lam.

LAURACEAE

Ocotea pulchella Mart. LEGUMINOSAE

*Canavalia rosea (Sw.) DC.

Centrosema virginianum (L.) Benth.

Crotalaria anagyroides H.B.K.

*Desmodium incanum DC.

Senna corymbosa (Lam.) Irwin et Barneby

Sophora tomentosa $\mathrm{L}$.

Stylosanthes viscosa $\mathrm{Sw}$.

LYTHRACEAE

Cuphea carthagenensis (Jacq.) Macbride MELASTOMATACEAE

Miconia sp.

Er

Er

Er

Er

Er

Er

Ar

Er

Er

Et

Er

Ar

Ar

Er

Er

Er

Ar

Er

Ar

Ar

Ar

Ar

Ar

Ar

Ar

Ar

Ar

Ar

Ar

Ar

Er

Ep

Er

Er

Ep

Er
$\mathrm{CH}$

$\mathrm{CA} / \mathrm{CH}$

$\mathrm{CH}$

$\mathrm{CH}$

$\mathrm{CH}$

$\mathrm{CH}$

CA

$\mathrm{CH}$

$\mathrm{CH}$

CA

$\mathrm{CH}$

CA

CA

$\mathrm{CH}$

$\mathrm{CH}$

CA

$\mathrm{CH} / \mathrm{CA}$

$\mathrm{CH}$

CA

CA

CA

CA

CA

CA

CA

CA

CA

CA

CA

CA

$\mathrm{CH}$

CA

CA

$\mathrm{CH}$

CA

$\mathrm{CH}$

*Oralis sp. 
Florística e estrutura da comunidade herbácea...

Tabela 1: Continuacão

Passiflora edulis Sims PIPERACEAE

Peperomia corcovadensis Gardner

PLANTAGINACEAE

* Plantago catharinea Decne.

$P$. paralias Decne.

POLYGALACEAE

${ }^{*}$ Polygala cyparissias St.-Hil.et Moquin

*P. molluginifolia St.-Hil

Et

CA

$\mathrm{Er} / \mathrm{Ep}$

CA

Er

$\mathrm{CH}$

Er

$\mathrm{CH}$

Er

$\mathrm{CH}$

Er

$\mathrm{CH}$

POLYPODIACEAE

Microgramma vacciniifolia (Langsd.et Fisch.) Copel.

Polypodium catharinae Langsd.et Fisch.

$* P$. lepidopteris (Langsd.et Fisch.)Kze.

PRIMULACEAE

*Anagallis arvensis $\mathrm{L}$.

RUBIACEAE

Chiococca alba (L.)Hitchcock

*Hedyotis salzmannii (DC.)Steud.

Psychotria Kleinii Smith et Downs

*Richardia brasiliensis Gomes

SAPINDACEAE

*Dodonaea viscosa (L.) Jacq.

SAPOTACEAE

Bumelia obtusifolia Roem. et Schult.

SCROPHULARIACEAE

*Linaria canadensis(L.) Dum.

SMILACACEAE

*Smilax campestris Griseb.

S. elastica Griseb.

SOLANACEAE

Petunia sp.

Solanum americanum Mill.

TILIACEAE

Triumfetta sp.

UMBELIFERAE

*Apium leptophyllum (Pers.) F.Muell

*Centella asiatica (L.)Urban

*Hydrocotyle bonariensis Lam.

VERBENACEAE

Lantana camara $\mathrm{L}$.

XYRIDACEAE

*Xyris sp.

Er: erva terrícola; Ep: erva epifítica; Et: erva trepadeira; Ar: arbusto; CA: comunidade arbustiva; $\mathrm{CH}$ : comunidade herbácea; *: espécies amostradas na vegetação herbácea. 
Tabela 2: Parâmetros Fitossociológicos das espécies amostradas na vegetação herbácea de dunas da Praia do Ferrugem, Garopaba-SC.

\begin{tabular}{lrllrlllr}
\hline ESPÉCIES & \multicolumn{1}{c}{$\mathrm{P}$} & $\mathrm{F}$ & $\mathrm{F} \%$ & \multicolumn{1}{c}{$\mathrm{C}$} & $\mathrm{C} \%$ & $\mathrm{D}$ & \multicolumn{1}{c}{$\mathrm{D} \%$} & $\mathrm{IVI}$ \\
\hline Mariscus pedunculatus & 29 & 0.58 & 8.21 & 9.3 & 3.02 & 5.40 & 16.54 & 27.77 \\
Spartina ciliata & 26 & 0.52 & 7.36 & 5.3 & 1.72 & 2.75 & 8.42 & 17.50 \\
Smilax campestris & 16 & 0.32 & 4.53 & 9.0 & 2.92 & 2.87 & 8.79 & 16.24 \\
Hydrocotyle bonariensis & 34 & 0.68 & 9.63 & 2.4 & 0.78 & 1.62 & 4.96 & 15.37 \\
Dodonaea viscosa & 8 & 0.16 & 2.27 & 14.6 & 4.73 & 2.34 & 7.17 & 14.17 \\
Evolvulus pussillus & 11 & 0.22 & 3.12 & 10.5 & 3.40 & 2.32 & 7.11 & 13.63 \\
Androtrichum trigynum & 16 & 0.32 & 4.53 & 6.6 & 2.14 & 2.11 & 6.46 & 13.13 \\
Polygala cyparissias & 27 & 0.54 & 7.65 & 2.2 & 0.71 & 1.16 & 3.55 & 11.91 \\
Eragrostis cataclasta & 1 & 0.02 & 0.28 & 30.0 & 9.73 & 0.60 & 1.84 & 11.85 \\
Senecio crassiflorus & 12 & 0.24 & 3.40 & 4.6 & 1.49 & 1.11 & 3.40 & 8.29 \\
Porophyllum ruderale & 19 & 0.38 & 5.38 & 1.9 & 0.62 & 0.71 & 2.17 & 8.17 \\
Imperata brasiliensis & 3 & 0.06 & 0.85 & 13.6 & 4.41 & 0.82 & 2.51 & 7.77 \\
Axonopus sp. & 14 & 0.28 & 3.97 & 2.4 & 0.79 & 0.68 & 2.08 & 6.84 \\
Andropogon selloanus & 1 & 0.02 & 0.28 & 15.0 & 4.86 & 0.30 & 0.92 & 6.06 \\
Desmodium incanum & 1 & 0.02 & 0.28 & 15.0 & 4.86 & 0.30 & 0.92 & 6.06 \\
Ipomoea pes-caprae & 1 & 0.02 & 0.28 & 15.0 & 4.86 & 0.30 & 0.92 & 6.06 \\
Juncus capillaceus & 1 & 0.02 & 0.28 & 15.0 & 4.86 & 0.30 & 0.92 & 6.06 \\
Noticastrum acuminatum & 1 & 0.02 & 0.28 & 15.0 & 4.86 & 0.30 & 0.92 & 6.06 \\
Polypodium lepidopteris & 1 & 0.02 & 0.28 & 15.0 & 4.86 & 0.30 & 0.92 & 6.06 \\
Richardia brasiliensis & 1 & 0.02 & 0.28 & 15.0 & 4.86 & 0.30 & 0.92 & 6.06 \\
Symphyopappus casarettoi & 1 & 0.02 & 0.28 & 15.0 & 4.86 & 0.30 & 0.92 & 6.06 \\
Ischaemum minus & 5 & 0.10 & 1.42 & 7.3 & 2.37 & 0.73 & 2.24 & 6.03 \\
Ipomoea littoralis & 9 & 0.18 & 2.55 & 3.5 & 1.13 & 0.33 & 1.93 & 5.61 \\
Oxypetalum tomentosum & 12 & 0.24 & 3.40 & 1.7 & 0.55 & 0.42 & 1.29 & 5.24 \\
Panicum sp. & 6 & 0.12 & 1.70 & 5.0 & 1.62 & 0.60 & 1.84 & 5.16 \\
Outras 41 espécies & 97 & 1.94 & 27.43 & 58.5 & 18.90 & 3.67 & 10.27 & 56.60 \\
TOTAIS & 353 & 7.06 & 99.92 & 308.4 & 99.91 & 32.64 & 99.93 & 299.76 \\
\hline & & & & & & & &
\end{tabular}

P: presença nas amostras; F: freqüência absoluta; F\%: freqüência relativa; C: cobertura média; C\%: cobertura relativa; D: densidade absoluta; D\%: densidade relativa e IVI: índice de valor de importância. 


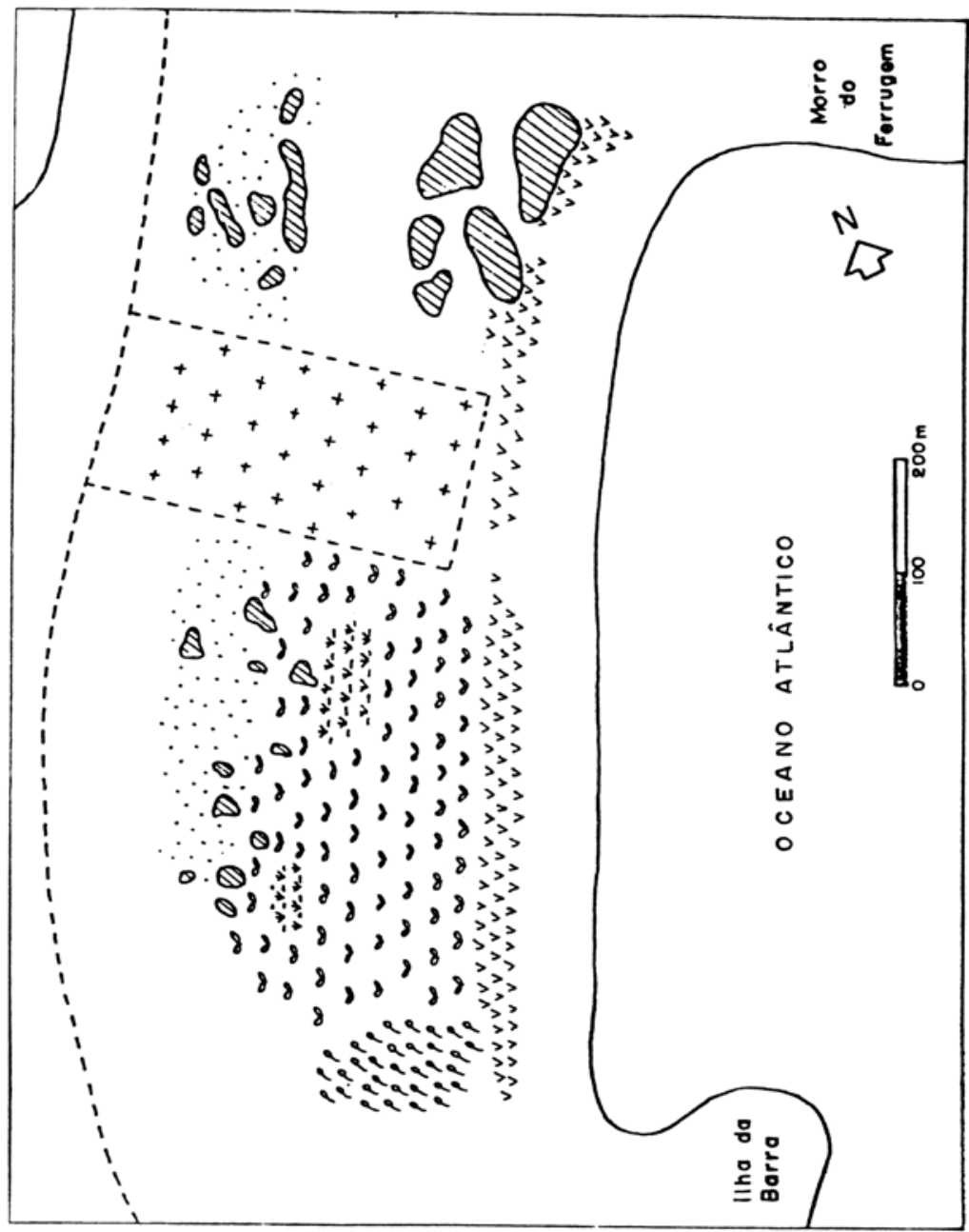

[. Vegetaçáo Halófila das Dunas Frontais

Pag Vegetação Psamófila das Dunas Móveis

¿: Vegetacão Psamófila das Dunas Estáveis

[... Vegetaçāo Psamófila das Baixadas Umidas

एT] Formacóes Arbustivas

Campos Litorâneos

${ }_{+}^{+}$Área Alterada

Figura 2 - Mapeamento da Vegetação da Praia do Ferrugem, SC. 
DANILEVICZ et al.

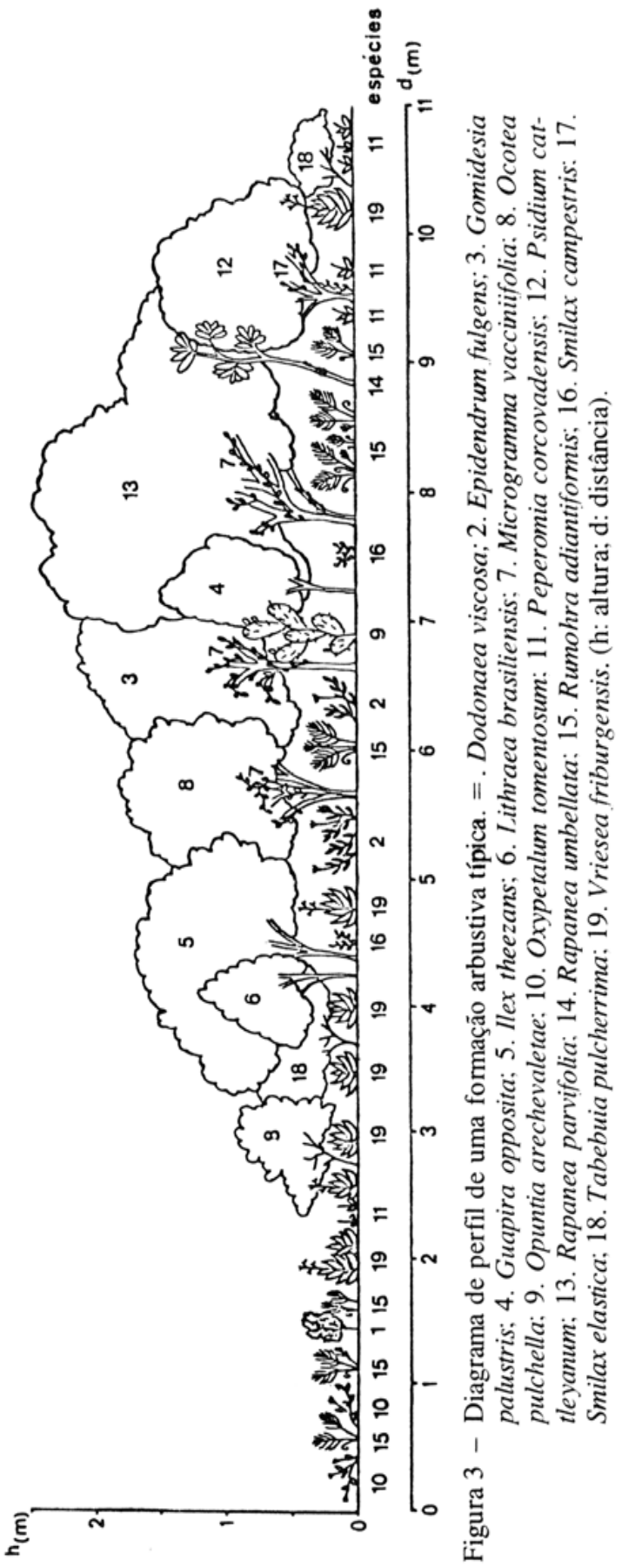

